\title{
Symptoms of the naturalisation of the Turkey oak (Quercus cerris L.) in Polish forests
}

\author{
Władysław Danielewicz $\bowtie$, Piotr Kiciński, Blanka Wiatrowska \\ Poznań University of Life Sciences, Department of Forest Botany, Wojska Polskiego 71 D, 60-625 Poznań, Poland, \\ phone: +48 618487746, e-mail: danw@up.poznan.pl
}

\begin{abstract}
The Turkey oak (Quercus cerris L.), the natural range of which embraces southern Europe and Asia Minor, belongs to trees rarely introduced into Polish forests. Tree stands where it appears, established before the Second World War, can be found in some 20 localities, mostly in the western part of the country. Because this species is capable of a natural renewal in a woodland environment, a research was made to find in what conditions and how far it undergoes spontaneous naturalisation. Three study sites were chosen in the forests of central Wielkopolska. An inventory was made of mature stands of the Turkey oak and its generative renewal. Plant communities in which the young generation of $Q$. cerris usually appears were characterised. It was found that self-sown seedlings of this species grew at a distance of up to $2,500 \mathrm{~m}$ from parent trees. The highest number and the greatest density of specimens of the secondary generation of the Turkey oak were found at 'Racot', which is a 100-hectare, mid-field woodland island where mesotrophic habitats predominate and where about $50 \%$ of the area is occupied by communities with manmade pine tree stands. At all sites, Q. cerris penetrates primarily this type of deformed phytocoenoses, developing mostly on former farmland. It has become a permanent component of the underbrush and undergrowth in them, and in some places, it also makes up the tree layer. It was observed that in the study area, it penetrated the woodland environment much more effectively than Quercus rubra, considered an invasive species. The expansion of the Turkey oak in several of the examined localities can be regarded as a basic manifestation of its naturalisation in places where there are phytocoenoses with pine stands in broad-leaf forest habitats in the neighbourhood of parent trees.
\end{abstract}

\section{KeY WORDS}

alien tree species, introduction, establishment, regeneration dynamics, dispersal

\section{INTRODUCTION}

The Turkey oak (Quercus cerris L.) is an important forest tree in the countries of southern Europe and Asia Minor, where it occurs in a natural state: from southern France, through Italy, Switzerland, Austria, the Balkan Peninsula, to Lebanon (Meusel et al. 1965; Browicz
1982; Menitski 1984; Bozano and Turok 2003). Outside its natural range, it is mostly cultivated as a fast-growing ornamental tree with modest soil requirements and a strong resistance to drought. In a few regions, especially in north-western France and southern Great Britain, it has been found in spontaneously developed secondary plant communities (Jalas and Suominen 1976; Preston et 
al. 2002). Attempts at an introduction of the Turkey oak to Polish forests for productive purposes have failed. The introduction was probably only intended to test the acclimatisation properties of the species, rather than its use at an economic scale because no research was made on the growth dynamics and productivity of the trees. Possibly, even preliminary observations of the frost damage of its tree trunks caused the range of the experimental cultivation of the Turkey oak in forests to be restricted (Danielewicz et al. 2014). Only fragments of tree stands containing this species have survived until today, mostly those established before the Second World War and usually on the land formerly belonging to the Prussian sector of divided Poland. Even so, it merits interest as one of the plants permanently established in Poland, and at a local scale, it may prove to be an invasive species requiring the control of its population dynamics in the wild (Danielewicz and Maliński 2003; Tokarska-Guzik et al. 2012). A summary of basic information about the 22 localities of the Turkey oak in Polish forests can be found in Danielewicz et al. (2014). Those authors observed symptoms of its spontaneous regeneration from seeds in almost all places where this species was represented in a mature tree stand by a dozen or more trees. However, it was not everywhere that further development of selfsown seedlings of $Q$. cerris and its penetration into forest communities was recorded.

The research reported in this paper was intended to find in what conditions the naturalisation of the Turkey oak took place in the woodland environment of selected sites in Poland, and how advanced is this process. The aim of the research was to document symptoms of this process, determined on the basic of properties in the local, secondary population of $Q$. cerris, such as the spatial range of spontaneous proliferation, abundance, density, and diversity of the size of individuals, which are formed by natural, generative regeneration, as well as a part of this tree in the structure and floristic composition of plant communities in which it occurs most frequently.

\section{MAterial AND MEthodS}

\section{Study sites}

Out of the 22 localities of the Turkey oak found in Polish forests (Danielewicz et al. 2014), 3 sites were chosen for study, in which this species was observed to dis- play the greatest ability for spontaneous dispersal away from places where seed-bearing trees grow. They all lie in central Wielkopolska (Fig. 1), from 20 to $47 \mathrm{~km}$ in a straight line from the region's capital, the city of Poznań. They were designated by the names of the forest districts where they are situated.

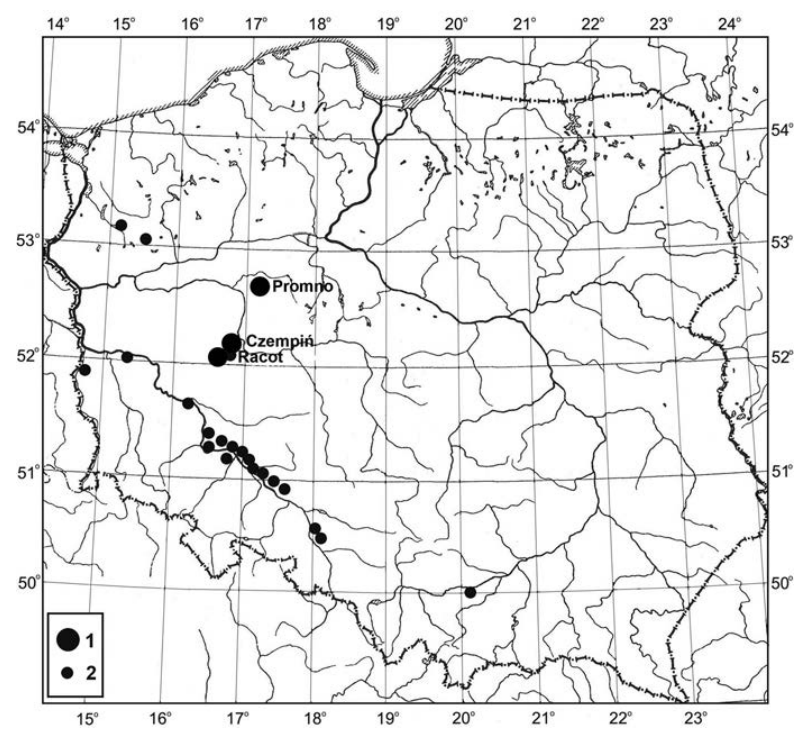

Figure 1. Location of study plots (1) relating to other Quercus cerris localities in forests of western Poland (2)

Site 1: 'Racot' in the Kościan Forest Inspectorate. The meso-region of the Leszno Upland. A small woodland area of 105 ha, surrounded by agricultural land. Divisions 197-201. The highest proportion of the woodland area is occupied by fertile and moderately fertile habitats: a fresh deciduous forest (Lśw), 50\%, and a mixed fresh forest (LMśw), 36\% (according to the classification of forest habitats (forest site type), used by the Polish foresters (Kliczkowska et al. 2004)). Half of the area is occupied by anthropogenic communities with Pinus sylvestris, the rest being mostly broad-leaf forests (of the oak-hornbeam forest type) with Quercus robur, less frequently with Acer pseudoplatanus, as well as phytocoenoses with Q. rubra, Pseudotsuga menziesii, Picea abies, and Larix decidua. The area with old-growth stands of Q. cerris, 123 years old (division $199 \mathrm{~g}$ ) is $0.82 \mathrm{ha}$.

Site 2: 'Czempiń' in the Konstantynowo Forest Inspectorate. The meso-region of the Leszno Upland. A small woodland area of 41 ha, surrounded by agricultural land. Divisions 261 and 262. Predominant habi- 
tats of fertile broadleaf forests: a fresh deciduous forest, $50 \%$, a moist forest, $39 \%$, while the proportion of mesotrophic habitats of a fresh mixed forest is a mere $9 \%$. Tree stands with predominantly broad-leaf species (Quercus robur, Fraxinus excelsior, Fagus sylvatica, and Alnus incana) occupy $78 \%$ of the woodland area, and those with the pine, $19 \%$. The greatest cluster of 25 Turkey oaks, over 120 years old, occurs in a multispecies broad-leaf tree stand in division $261 \mathrm{~b}$.

Site 3: 'Promno' in the area of the Czerniejewo Forest Inspectorate. The mesoregion of Wielkopolska Lakelands. Its north-western fragment, called the Czerniejewo Forests, is a large woodland island occupying $423 \mathrm{ha}$, surrounded by agricultural land, and in the north-east neighbouring on the village of PobiedziskaLetnisko. Divisions 215-231. The highest proportion of the woodland area, $48 \%$, is occupied by a fresh mixed deciduous forest, and $28 \%$ by a fresh deciduous forest. The habitats of a fresh mixed coniferous forest account for $7 \%$ of the woodland area, and a fresh coniferous forest, $2 \%$. Stands of Pinus sylvestris occupy $47 \%$ of the area, Quercus sp. 35\%, Alnus sp. 12\%, and other species as Fagus sylvatica, Carpinus betulus, Fraxinus excelsior, or Populus tremula $-6 \%$. The area with 61 oldgrowth stands of Q.cerris, 122 years old (division 229 a) is 0.40 ha.

\section{Structure of the thickness of the oldest stands of the Turkey oak}

Measurements of tree thickness were taken in 2015 in the oldest stands of $Q$. cerris in the case of which there was no doubt that they had been planted. It was only at 'Promno' that such measurements were also performed many years earlier, in 1967 (Juwa 1968), hence only in this case was it possible to present the structure of the stand in the period preceding the substantial decline in the number of the trees. Because of the scattering of the oldest planted Turkey oaks over a few places at 'Czempin', the structure of the tree stand with this species at this site is not presented in the article.

\section{Distribution and structure of the natural regeneration of the Turkey oak}

This part of the study was conducted at 'Racot' and 'Promno'. No such work was carried out at 'Czempiń' because of the concentration of the undoubtedly spontaneous regeneration of the Turkey oak in only one place at a distance from the parent tree stand. Besides, the origin of trees of this species in a few sapling stands in this area is not clear. They may have been planted together with native oaks, although it is possible that they have appeared there spontaneously.

The search for the natural regeneration of Quercus cerris embraced all areas with old-growth stands of this species as well as forests situated in their neighbourhood. No study was made of the regeneration of the Turkey oak outside forests, for example, at roadsides and on railway embankments. The position of each specimen coming from a self-sown seedling was determined in terms of geographical coordinates using Trimble Juno 3B GPS equipment. At 'Racot', where there is a mature stand of Q. rubra, young specimens of this species were also recorded. Because of changes that have recently occurred as a result of forest husbandry in division $198 \mathrm{f}$ (tree cutting and artificial forest regeneration) at the 'Racot' site, use was made of materials collected in 2006.

Measurements were made of the height of the oaks forming a natural regeneration (with the exception of one-year-old seedlings) and their diameters at an altitude of $1.3 \mathrm{~m}$, if greater than $5 \mathrm{~cm}$.

\section{Plant communities with the Turkey oak}

At all sites accordance with the methods used in phytosociology, relevés were made in places with mature tree stands containing the Turkey oak (15 relevés) and in communities where this species had appeared spontaneously and now covers at least $5-25 \%$ of the area (24 relevés). Also, in this case, relevés in division $198 \mathrm{f}$ come from the year 2006 for the reason explained earlier.

\section{Results}

\section{Thickness structure of the oldest stands with the Turkey oak}

It can be assumed that the only source of seeds through which the Turkey oak could disperse over the study area was tree stands containing this species established at the end of the 19th and the beginning of the 20th centuries that attained generative maturity some 50-60 years ago. At each site, there is one such stand outside of which not even single trees planted at that time were 
found. The probability that they grew in other places, but were overlooked, or that they had been cut down before the research began, is very low.
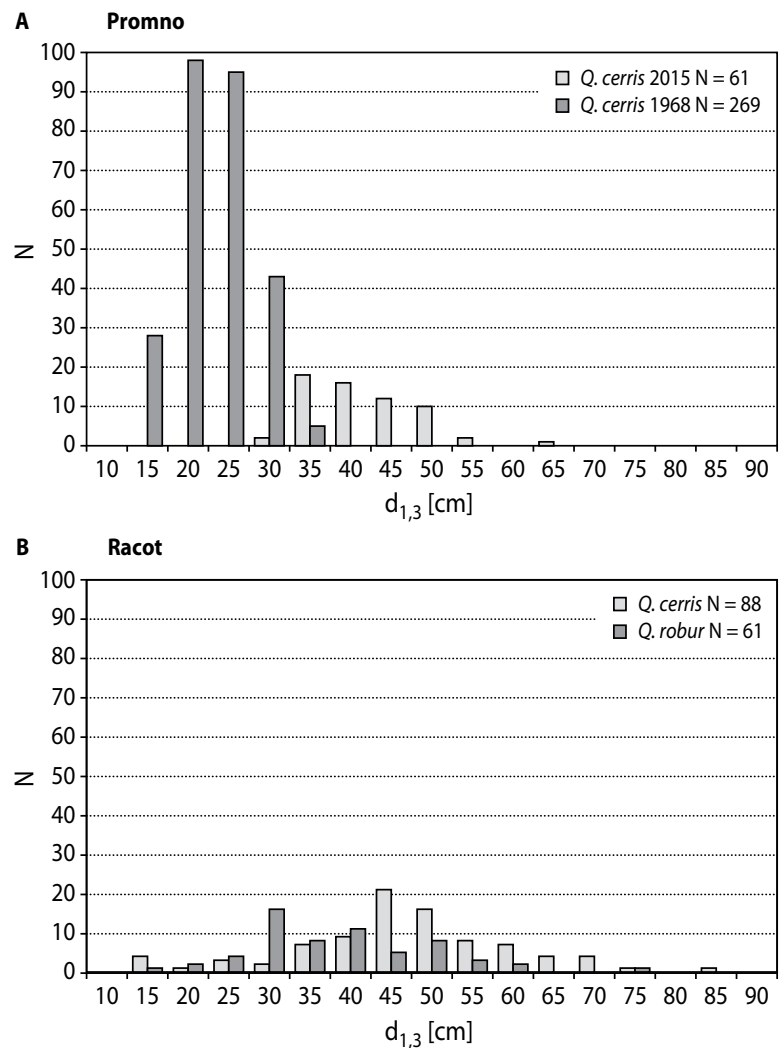

Figure 2. Thickness structure of the oldest stands of Quercus cerris and Q. rubra - other alien tree from the genus Quercus, which spreads in the same area

In 1967, there were 269 introduced Turkey oaks at 'Promno', which means that more than 200 trees had disappeared over the nearly 50 years since then. Because there are no detailed data on how this number kept declining, one can only suppose that the reduction was mostly due to tending cuts, which is reflected in the distribution of breast-high diameters characteristic of managed tree stands (Fig. 2A). In the mixed stand of Quercus cerris and Q. roburat 'Racot', the range of tree thickness is greater and the distribution closer to normal (Fig. 2B), which may be connected with tending cuts being less intensive here. At both sites, however, the Turkey oak stands established over 100 years ago have gone through all development stages and attained generative maturity at least a few decades ago.

\section{Distribution and structure of the natural regeneration of the Turkey oak}

The most exuberant natural regeneration of the Turkey oak was recorded at 'Racot', with 1,516 specimens from self-sown seedlings growing at a distance from

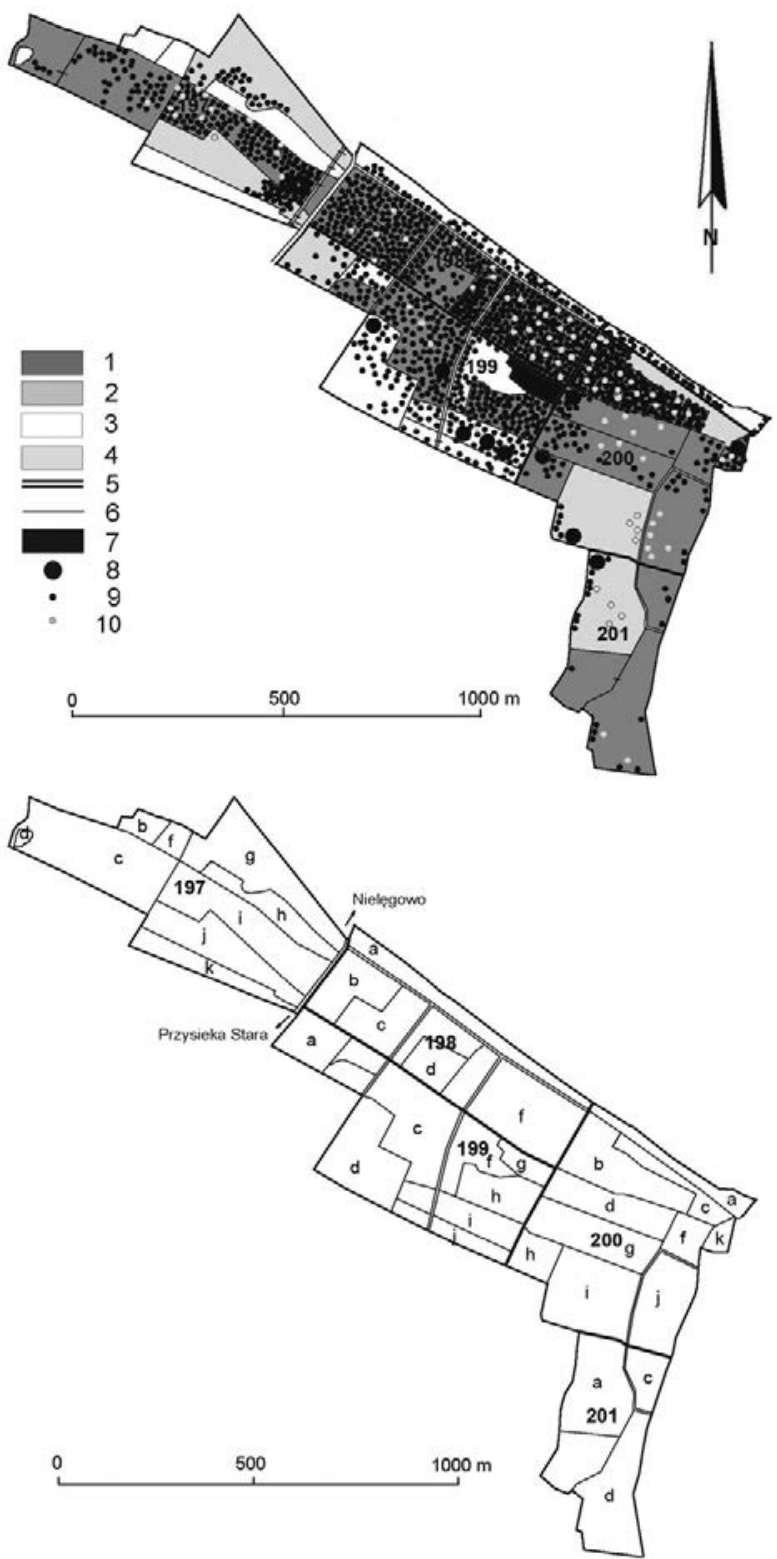

Figure 3. Distribution of localities of the Turkey oak at 'Racot' forest: 1 - Pinus sylvestris stands, 2 -Quercus robur or Q. petraea stands, 3 - other broad-leaf stands, 4 - non-forest environment, 5 - roads, 6 - forest subsection boundaries, 7 - oldest $Q$. cerris stands, 8 - young plantation of $Q$. cerris, 9 - natural regeneration of $Q$. cerris, 10 - natural regeneration of $Q$. rubra 

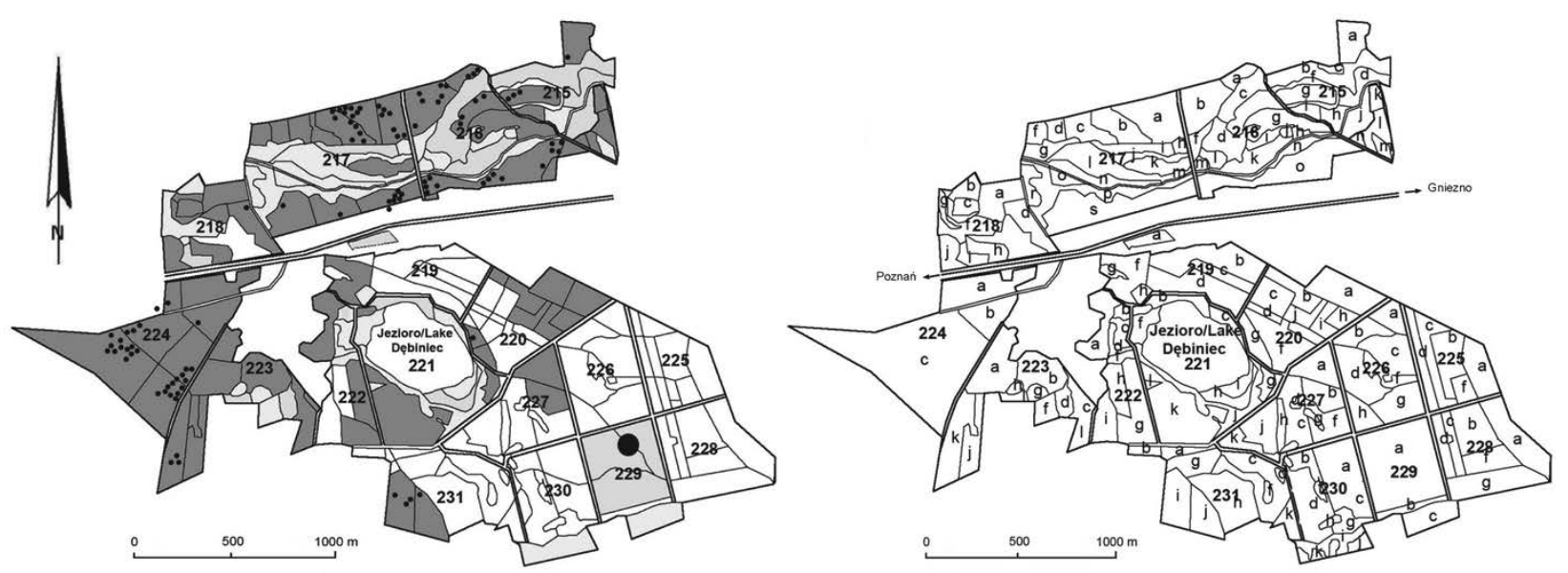

Figure 4. Distribution of localities of the Turkey oak at 'Promno' (for description see Fig. 3)

the parent tree stand. They can mostly be found in the north-eastern parts of divisions 198 and 200, fragments of division 199 near the old Q. cerris trees, and the part of division 197 farthest from them (Fig. 3). In the young natural regeneration and undergrowth, a much rarer species is $Q$. rubra (108 specimens), the distribution of which is presented in Figure 2, which indicates that it plays a decidedly lesser role than $Q$. cerris in the penetration of the woodland environment at this site. It occurs most frequently in the places of exuberant regeneration of the Turkey oak (divisions: 198f, 200b).

The maximum distance between the parent stand of $Q$. cerris and specimens of its natural regeneration at 'Racot' is $1,468 \mathrm{~m}$, close to the border of the woodland area. At 'Promno', where the regeneration is much less exuberant (102 specimens) and located much farther from the old trees, the distance extends to 2,460 $\mathrm{m}$ (Fig. 4). A common feature in the distribution of the secondary localities of $Q$. cerris that can be observed on the maps of those two sites (Fig. 3 and 4) is that most of them are located in forests with pine tree stands ('Racot') or exclusively in such forests ('Promno').

The highest density of the natural regeneration of the Turkey oak at 'Racot' is almost 100 specimens/ha in division 200b (fresh mixed coniferous forest [BMśw], a pine tree stand aged 87), located in the close vicinity of an old-growth stand of this species (Tab. 1). There were $50(3 \%)$ Q. cerris specimens lower than $1 \mathrm{~m}$ at this site and $757(50 \%)$ specimens with a breast-high diameter of under $5 \mathrm{~cm}$ (Fig. 5 and 6). The lowest Turkey oaks coming from natural regeneration grow farthest away from the parent stand (division 197), and in division 201, where their number is the smallest. In divisions 198 and 200, where the number and density of the secondary generation of the oak are the highest, $70 \%$ and $79 \%$ trees, respectively, were part of the undergrowth, in some cases of the young natural regeneration, and the rest, $30 \%$ and $21 \%$, reached the lower tree layer. With a lower natural regeneration rate in division 199 , the proportion of specimens in the tree layer is much higher, at 74\%. At 'Promno', the predominant height of Q. cerris is $1-5 \mathrm{~m}(86 \%)$, with only 9 trees (8\%) higher than $5 \mathrm{~m}$.

Table 1. Number of Turkey oak specimens from natural regeneration per ha at "Racot"

\begin{tabular}{|l|c|c|c|c|c|c|}
\hline \multirow{2}{*}{$\begin{array}{l}\text { Forest } \\
\text { sections and } \\
\text { subsections } \\
\text { (area in ha) }\end{array}$} & \multicolumn{3}{|c|}{ Number of individuas in forest subsections } \\
\cline { 2 - 7 } & min. & max. & total & min. & max. & $\begin{array}{c}\text { aver- } \\
\text { age }\end{array}$ \\
\cline { 2 - 7 } $\begin{array}{l}197 \mathrm{f}-\mathrm{k} \\
(27,07)\end{array}$ & $22(\mathrm{i}, \mathrm{k})$ & $185(\mathrm{j})$ & 305 & $6(\mathrm{k})$ & $41(\mathrm{j})$ & 11 \\
\hline $\begin{array}{l}198 \mathrm{a}-\mathrm{f} \\
(18,18)\end{array}$ & $19(\mathrm{~d})$ & $161(\mathrm{f})$ & 401 & $13(\mathrm{~d})$ & $25(\mathrm{f})$ & 21 \\
\hline $\begin{array}{l}199 \mathrm{a}-\mathrm{f}, \mathrm{h}-\mathrm{j} \\
(21,37)\end{array}$ & $11(\mathrm{a}, \mathrm{j})$ & $88(\mathrm{~h})$ & 293 & $5(\mathrm{a})$ & $51(\mathrm{~h})$ & 14 \\
\hline $\begin{array}{l}200 \mathrm{a}-\mathrm{b}, \mathrm{d}-\mathrm{k} \\
(24,01)\end{array}$ & $5(\mathrm{i})$ & $370(\mathrm{~b})$ & 499 & $1(\mathrm{i})$ & $97(\mathrm{~b})$ & 21 \\
\hline $\begin{array}{l}201 \mathrm{a}, \mathrm{c}-\mathrm{f} \\
(11,44)\end{array}$ & $2(\mathrm{f})$ & $8(\mathrm{a})$ & 18 & $1(\mathrm{~d})$ & $3(\mathrm{f})$ & 2 \\
\hline
\end{tabular}

The spatial differences in the size of Turkey oaks from natural regeneration at 'Racot' are also reflected 

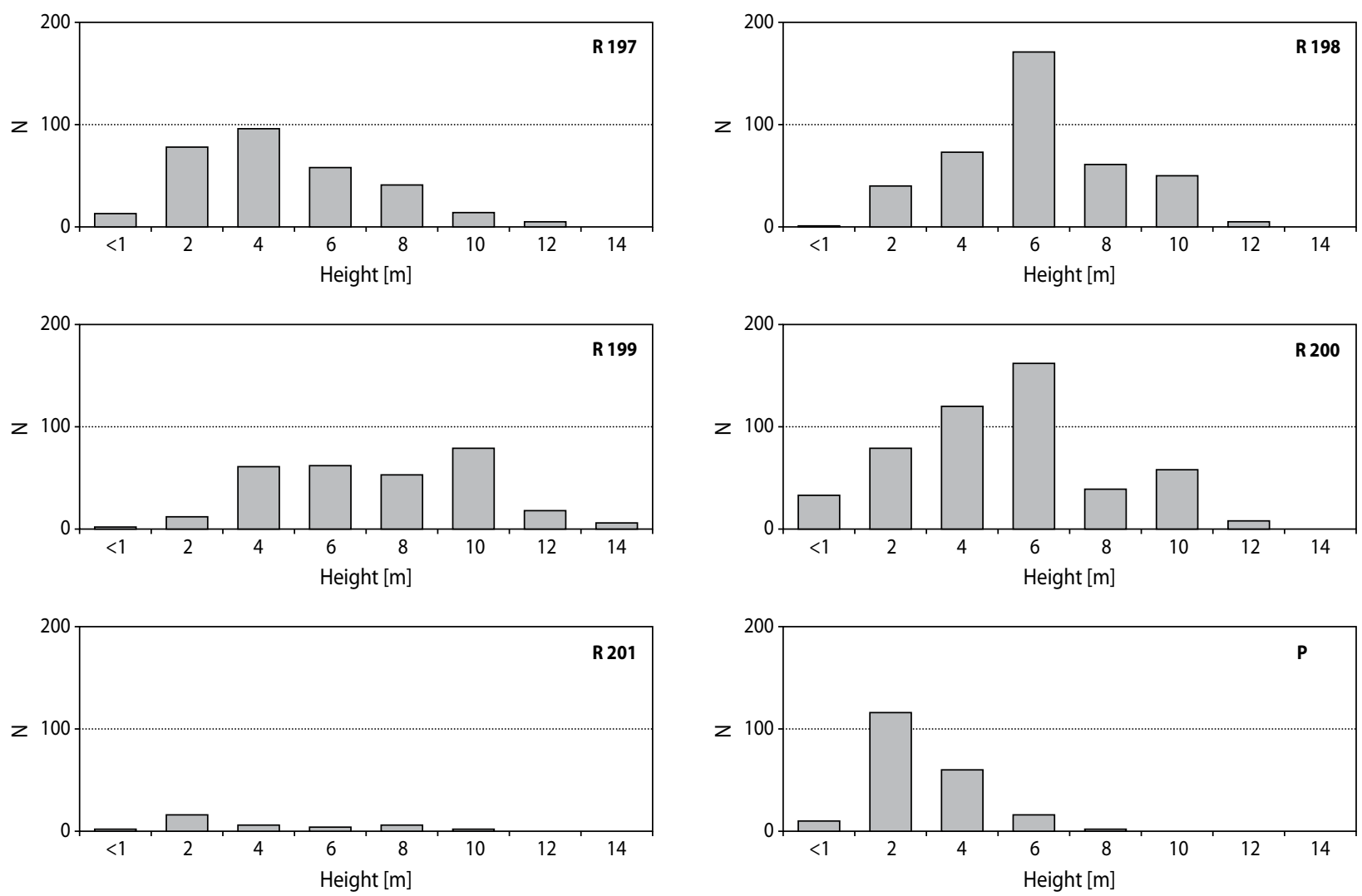

Figure 5. Differences in the height of the Turkey oak from natural regeneration at 'Racot' (R) and 'Promno' (P), number of forest sub-compartment

in the distribution of their thickness (Fig. 6). In four divisions: 197, 198, 200, and 201, the highest proportion is that of oaks with a breast-high diameter of under $5 \mathrm{~cm}$ (including those that have not attained the height of $1.3 \mathrm{~m}$ ): $52 \%, 68 \%, 50 \%$, and $56 \%$, respectively. Their proportion in division 199 is the smallest, at 23\%. Apart from division 201, where there are only 18 young specimens, the most numerous are trees 5-9 cm thick, but in division 199, their proportion in the number of all trees with a breast-high diameter of $5 \mathrm{~cm}$ and more amounts to $45 \%$, markedly lower than in the other divisions (197-73\%, 198-63\%, and 200-60\%). At 'Promno' as a whole, Turkey oaks with the smallest thickness values predominate.

\section{Communities with the Turkey oak}

At all the study sites, phytocoenoses with the old growth of the Turkey oak occurred in areas where the potential association in fresh deciduous forest habitats is the Central-European oak-hornbeam forest Galio
sylvatici-Carpinetum, but the actual vegetation is dominated by communities deformed by forest husbandry. Also, the phytocoenoses under study differ considerably from the natural oak-hornbeam communities in terms of structure and floristic composition (Tab. 2). Tree stands are usually oak monocultures developed in accordance with the principles of forest cultivation. It is only at 'Promno' that Carpinus betulus and Fagus sylvatica are an admixture. The proportion of forest ground-layer plants typical of fertile and moderately fertile broad-leaf forests is small. What distinguished the 'Promno' and 'Czempin' phytocoenoses is only a slightly greater proportion of species characteristic of the class Querco-Fagetea. A symptom of the advanced degeneration of the examined communities with the Turkey oak is a substantial representation of the class Artemisietea, and at 'Racot', a markedly higher contribution of species of the class Epilobietea. In many places of this site, Rubus sp. forms lush clusters; hence, a high proportion of forest phytocoenoses show 

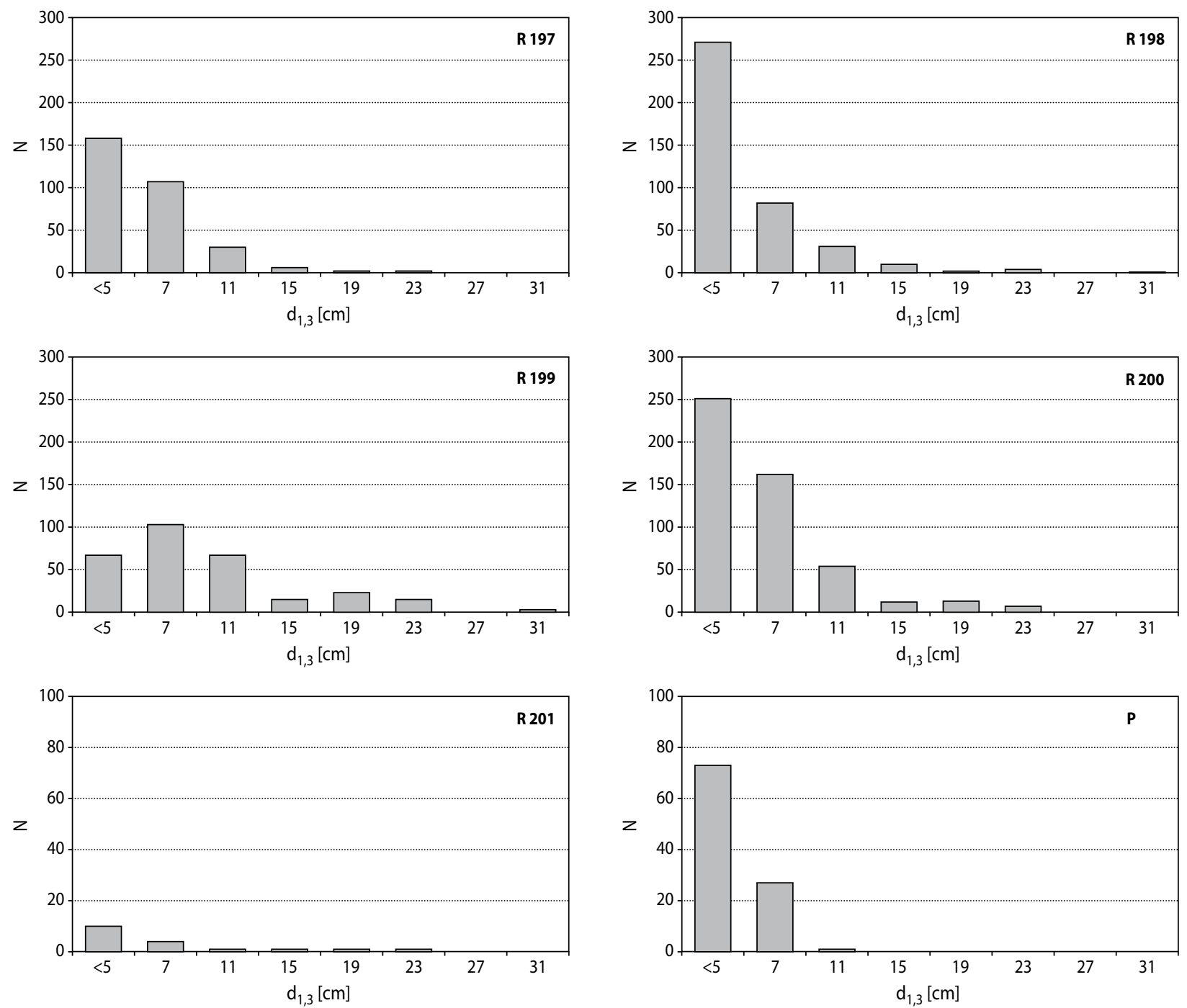

Figure 6. Differences in the thickness of the Turkey oak from natural regeneration at 'Racot' (R) and 'Promno' (P)

signs of so-called rubietisation, one of the six forms of forest communities degeneration distinguished by Olaczek $(1972,1974)$, involving mass development of those plants. What is characteristic here is a more frequent occurrence of $Q$. cerris in the undergrowth and in the herbal layer.

Table 2. Communities with old-growth Turkey oak

\begin{tabular}{|c|c|c|c|c|c|c|c|c|c|c|c|c|c|c|c|c|}
\hline \multicolumn{2}{|c|}{ Successive No } & 1 & 2 & 3 & 4 & 5 & 6 & 7 & 8 & 9 & 10 & 11 & 12 & 13 & 14 & 15 \\
\hline \multirow{2}{*}{ Date } & Day & 07 & 07 & 07 & 01 & 01 & 15 & 15 & 15 & 15 & 15 & 15 & 15 & 15 & 15 & 15 \\
& Month & 06 & 06 & 06 & 06 & 06 & 06 & 06 & 06 & 06 & 06 & 06 & 06 & 06 & 06 & 06 \\
& Year & 15 & 15 & 15 & 15 & 15 & 15 & 15 & 15 & 15 & 15 & 15 & 15 & 15 & 15 & 15 \\
\hline \multicolumn{2}{|l|}{ Density of a, a1* } & $60 *$ & 60 & 60 & 70 & 80 & 80 & 70 & 70 & 90 & 70 & 60 & 70 & 50 & 60 & 70 \\
\hline Density of a2 & 30 & & & & & & & & & & & & & & \\
\hline Density of b & 20 & 20 & 40 & 5 & 5 & 40 & 40 & 30 & 10 & 30 & 50 & 40 & 60 & 50 & 10 \\
\hline
\end{tabular}




\begin{tabular}{|c|c|c|c|c|c|c|c|c|c|c|c|c|c|c|c|}
\hline Successive $\mathrm{N}^{\circ}$ & 1 & 2 & 3 & 4 & 5 & 6 & 7 & 8 & 9 & 10 & 11 & 12 & 13 & 14 & 15 \\
\hline Cover of $\mathrm{c}$ & 80 & 90 & 60 & 90 & 70 & 70 & 80 & 70 & 80 & 60 & 40 & 70 & 80 & 90 & 80 \\
\hline Cover of $d$ & $<5$ & $<5$ & $<5$ & 5 & 5 & 5 & $<5$ & 10 & $<5$ & - & - & - & - & $<5$ & - \\
\hline Area $\left[\mathrm{m}^{2}\right]$ & 400 & 400 & 200 & 400 & 400 & 200 & 200 & 200 & 200 & 200 & 200 & 200 & 200 & 200 & 200 \\
\hline $\begin{array}{l}\text { Locality } \\
\text { Forest section }\end{array}$ & $\begin{array}{l}\mathrm{P} \\
2 \\
2 \\
9 \\
\mathrm{a}\end{array}$ & $\begin{array}{l}\mathrm{P} \\
2 \\
2 \\
9 \\
\mathrm{a}\end{array}$ & $\begin{array}{l}\mathrm{P} \\
2 \\
2 \\
9 \\
\mathrm{a}\end{array}$ & $\begin{array}{l}\mathrm{C} \\
2 \\
6 \\
1 \\
\mathrm{~b}\end{array}$ & $\begin{array}{l}\mathrm{C} \\
2 \\
6 \\
1 \\
\mathrm{~b}\end{array}$ & $\begin{array}{l}\mathrm{R} \\
1 \\
9 \\
9 \\
\mathrm{~g}\end{array}$ & $\begin{array}{l}\mathrm{R} \\
1 \\
9 \\
9 \\
\mathrm{~g}\end{array}$ & $\begin{array}{l}\mathrm{R} \\
1 \\
9 \\
9 \\
\mathrm{~g}\end{array}$ & $\begin{array}{l}\mathrm{R} \\
1 \\
9 \\
9 \\
\mathrm{~g}\end{array}$ & $\begin{array}{l}\mathrm{R} \\
1 \\
9 \\
9 \\
\mathrm{~g}\end{array}$ & $\begin{array}{l}\mathrm{R} \\
1 \\
9 \\
9 \\
\mathrm{~g}\end{array}$ & $\begin{array}{l}\mathrm{R} \\
1 \\
9 \\
9 \\
\mathrm{~g}\end{array}$ & $\begin{array}{l}\mathrm{R} \\
1 \\
9 \\
9 \\
\mathrm{~g}\end{array}$ & $\begin{array}{l}\mathrm{R} \\
1 \\
9 \\
9 \\
\mathrm{~g}\end{array}$ & $\begin{array}{l}\mathrm{R} \\
1 \\
9 \\
9 \\
\mathrm{~g}\end{array}$ \\
\hline Stand age & 122 & 122 & 122 & 123 & 123 & 107 & 107 & 107 & 107 & 107 & 107 & 107 & 107 & 107 & 107 \\
\hline Number of species & 26 & 34 & 23 & 37 & 31 & 27 & 23 & 27 & 20 & 17 & 16 & 25 & 21 & 20 & 20 \\
\hline \multicolumn{16}{|l|}{ Trees and shrubs } \\
\hline Quercus cerris a, a1* & $4.3^{*}$ & 4.3 & 4.4 & 2.2 & 2.2 & 4.4 & 3.2 & 2.2 & 1.1 & 2.2 & 3.2 & 3.2 & 3.2 & 3.3 & 4.3 \\
\hline Quercus cerris b & . & + & . & . & . & 3.2 & 2.2 & 2.2 & . & . & 2.2 & 2.2 & 1.1 & 2.2 & 2.2 \\
\hline Quercus cerris c & + & 1.1 & + & 1.2 & 1.2 & 3.3 & 2.2 & 2.2 & . & + & 2.2 & 2.2 & 2.2 & 2.2 & 2.2 \\
\hline Quercus robur a & . & . & . & 3.3 & 3.3 & 1.2 & 2.2 & . & 5.5 & 3.3 & 2.2 & 2.2 & . & . & 1.1 \\
\hline Quercus robur b & . & . & . & . & . & . & . & . & . & . & . & . & . & . & 1.1 \\
\hline Quercus robur c & . & . & . & . & . & . & . & . & . & . & . & + & . & + & + \\
\hline Robinia pseudoacacia a & . & . & . & . & . & 2.2 & 3.3 & . & . & . & . & 2.2 & . & 2.2 & . \\
\hline Robinia pseudoacacia b & . & . & . & . & . & 1.1 & . & . & . & . & 2.2 & & 4.4 & 3.3 & \\
\hline Robinia pseudoacacia c & . & . & . & . & . & . & . & + & . & . & . & + & & & \\
\hline Quercus rubra a & . & . & . & . & . & . & . & 3.2 & . & . & . & . & . & . & . \\
\hline Quercus rubra c & . & . & . & . & . & . & . & 2.2 & + & + & . & . & . & . & . \\
\hline Fagus sylvatica a1 & 1.1 & . & . & . & . & . & . & . & . & . & . & . & . & . & . \\
\hline Fagus sylvatica a2 & 2.2 & $\cdot$ & . & . & . & . & . & . & . & . & . & . & . & . & . \\
\hline Fagus sylvatica b & 2.2 & 2.1 & 3.2 & . & . & . & . & . & . & . & . & . & . & . & . \\
\hline Fagus sylvatica $\mathrm{c}$ & 1.1 & . & 1.1 & . & . & . & . & . & . & . & . & . & . & . & . \\
\hline Carpinus betulus a2 & 2.2 & . & . & . & . & . & . & . & . & . & . & . & . & . & . \\
\hline Carpinus betulus b & 2.2 & 1.1 & . & . & . & . & . & . & . & . & . & . & . & . & . \\
\hline Ulmus laevis a & . & . & . & . & . & . & . & . & . & 1.1 & . & . & . & . & . \\
\hline Ulmus laevis b & . & . & . & . &. & . & . & 1.1 & . & 2.2 & . & . & . & . & . \\
\hline Sorbus aucuparia b & . & . & . & . & . & 1.1 & . & . & 1.1 & . & 2.2 & 2.2 & . & . & . \\
\hline Sorbus aucuparia c & . & . & . & + & + & & + & + & 1.1 & . & + & 1.1 & + & & + \\
\hline Prunus serotina $\mathrm{b}$ & + & + & . & + & . & 1.1 & . & + & . & . & . & 1.1 & + & & 1.1 \\
\hline Prunus serotina c & . & . & . & . & + & . & + & . & . & . & . & . & . & + & \\
\hline Prunus padus $\mathrm{b}$ & . & . & . & + & . & 2.2 & 1.1 & . & . & . & 1.1 & 1.1 & . & 1.1 & . \\
\hline Prunus padus c & . & . & . & 1.2 & + & . & . & . & . & . & + & . & . & + & \\
\hline Acer pseudoplatanus b & & 1.2 & . & . & . & . & . & 1.1 & 2.2 & 2.2 & . & . & . & . & . \\
\hline Acer pseudoplatanus $\mathrm{C}$ & . & . & . & . & . & . & . & . & 1.1 & 2.2 & . & . & . & . & . \\
\hline Fraxinus excelsior b & . & . & . & 1.1 & . & . & . & . & . & . & . & . & . & . & . \\
\hline Fraxinus excelsior c & . & . & . & . & + & . & . & 1.1 & + & & . & . & . & . & . \\
\hline Pyrus pyraster b & . & . & . & . & . & . & . & . & . & . & + & . & . & . & . \\
\hline Pyrus pyraster c & . & . & . & . & . & + & . & + & . & + & + & . & . & . & . \\
\hline Crataegus monogyna $\mathrm{b}$ & . & . & . & . & . & . & . & . & . & 1.2 & & 1.2 & 1.2 & . & . \\
\hline Prunus spinosa $\mathrm{b}$ & . & . & . & . & . & . & . & 2.2 & 1.2 & . & & . & & & \\
\hline
\end{tabular}




\begin{tabular}{|c|c|c|c|c|c|c|c|c|c|c|c|c|c|c|c|}
\hline Successive $\mathrm{N}^{\circ}$ & 1 & 2 & 3 & 4 & 5 & 6 & 7 & 8 & 9 & 10 & 11 & 12 & 13 & 14 & 15 \\
\hline Crataegus monogyna c & . & . & . & + & . & . & . & . & . & +.2 & . & . & . & . & . \\
\hline Frangula alnus b & . & . & . & & . & + & . & & . & . & . & . & . & . & . \\
\hline Frangula alnus $\mathrm{c}$ & . & . & . & + & . & . & . & . & . & . & . & . & . & . & . \\
\hline Rhamnus cathartica b & . & . & . & 1.1 & . & . & . & . & . & . & . & . & . & . & . \\
\hline Rhamnus cathartica c & . & . & . & + & . & . & . & . & . & . & . & . & . & . & . \\
\hline Pinus sylvestris c & . & . & . & . & . & . & . & . & . & . & . & . & + & + & . \\
\hline Betula pendula $\mathrm{c}$ & . & . & . & . & . & . & . & . & . & . & . & . & + & + & . \\
\hline \multicolumn{16}{|l|}{ Ch. Querco-Fagetea } \\
\hline Poa nemoralis & 1.2 & 1.2 & 1.2 & 3.3 & 1.2 & 2.3 & 2.2 & 2.3 & 1.2 & 2.2 & 2.3 & 2.3 & 2.2 & . & 3.3 \\
\hline Atrichum undulatum $\mathrm{d}$ & 1.2 & 1.2 & 1.2 & 1.2 & 1.2 & 1.2 & 1.2 & 2.2 & 1.2 & . & . &. &. & 1.2 & . \\
\hline Dryopteris filix-mas & 2.2 & 2.2 & 1.2 & 1.2 & . & . & 2.2 & . & . & . & . & 1.2 & 2.2 & 1.2 & . \\
\hline Anemone nemorosa & 2.2 & 1.2 & 2.2 & . & . & . & . & . & . & . & . & . & . & . & . \\
\hline Milium effusum & 1.2 & 1.2 & 1.2 & . & . & . & . & . & . & . & . & . & . & . & . \\
\hline Brachypodium sylvaticum &. & 1.2 & . & 1.2 & 1.2 & . & . & . & . & . & . & . & . & . & . \\
\hline Scrophularia nodosa & . & 1.2 & . & 2.2 & + & . & . & . & . & . & . & . & . & . & . \\
\hline Viola reichenbachiana & + & + & . & 1.2 & . & . & . & . & . & . & . & . & . & . & . \\
\hline Circaea lutetiana & + & + & + & . & . & . & . & . & . & . & . & . & . & . & . \\
\hline Ajuga reptans & . & 2.2 & 1.2 & . & . & . & . & . & . & . & . & . & . & . & . \\
\hline Dactylis polygama & 1.2 & & . & . & 1.2 & . & . & . & . & . & . & . & . & . & . \\
\hline Carex sylvatica & 1.2 & 1.2 & . & . & & . & . & . & . & . & . & . & . & . & . \\
\hline Stachys sylvatica & 1.1 & + & . & . & & . & . & . & . & . & . & . & . & . & . \\
\hline \multicolumn{16}{|c|}{ Ch. Quercetaea robori-petraeae } \\
\hline Carex pilulifera & . & . & . & . & . & 1.2 & . & 1.2 & . & . & . & 1.2 & 1.2 & . & 2.2 . \\
\hline Pteridium aquilinum & . & . & 1.2 & . & . & . & . & . & 1.2 & 1.2 & . & . & . & . & . \\
\hline Veronica officinalis & . & . & . & 2.2 & 1.2 & . &. & . & . & . & . & . & . & . & . \\
\hline Holcus mollis & . & . & . & 1.2 & . & . & . & 1.2 & & . & . & . & . & . & . \\
\hline Dicranella heteromalla d & . & . & . & . & . & . & . & . & . & . & . & . & +.2 & 1.2 & . \\
\hline Polytrichum formosum $\mathrm{d}$ & . & . & . & . & . & + & . & 1.2 & . &. & . &. & . &. &. \\
\hline \multicolumn{16}{|l|}{ Ch. Epilobietea } \\
\hline Rubus apricus & . & . & . & . & . & 2.3 & 2.2 & 3.3 & 3.3 . & 1.2 & 1.2 & 3.3 & 3.3 & 4.5 & 2.3 \\
\hline Rubus seebergensis & . & . & . & . & . & 2.2 & 2.2 & 1.2 & 2.2 & 1.2 & 1.2 & 1.2 & 2.2 & 2.2 & 2.3 \\
\hline Rubus gracilis & . & 1.2 & . & . & . & 2.3 & 2.2 & 2.2 & 2.2 & 1.2 & . & 1.2 & 2.2 & 2.2 & 2.2 \\
\hline Calamagrostis epigeios & . & . & . & . & 2.2 & . & . & 2.3 & 2.3 & 1.2 & 2.3 & . & 1.2 & 2.3 & . \\
\hline Rubus macrophyllus & . & . &. & . & . & . & . & . & . & 1.2 & . & 1.2 & . & +.2 & . \\
\hline Rubus idaeus & + & . & 1.2 & 2.2 & . & . & . & . & . & . & . & . & . & . & . \\
\hline Rubus lamprocaulos & . & . & . & & & 1.2 & . & . & . & . & . & 1.2 & . & . & . \\
\hline Fragaria vesca & . & + & . & . & + & . &. & . & . & . & . &. & . & . & . \\
\hline \multicolumn{16}{|l|}{ Ch. Atremisietea } \\
\hline Moehringia trinervia & 1.1 & 1.2 & + & 1.2 & 2.2 & 1.2 & 1.2 & 1.2 & 1.2 & . & + & 1.2 & 1.2 & + & +.2 \\
\hline Impatiens parviflora & 3.4 & + & 3.3 & . & . & 2.3 & 3.3 & . & 2.2 & 3.3 & 2.3 & 2.2 & 3.3 & 2.3 & 2.3 \\
\hline Fallopia convonvulus & + & 1.2 & 1.2 & . & 1.2 & 1.2. & +.2 & 1.2 & 1.2 & . & . & 1.2 & 1.2 & +.2 & + \\
\hline Urtica dioica & 2.2 & 2.2 & 2.2 & 1.2 & 1.2 & . & +.2 & . & . & . & . & 2.2 & 1.2 & . & 2.2 \\
\hline Galeopsis pubescens & 2.1 & 1.2 & 2.2 & 1.2 & 1.1 & . & + & 1.2 & . & 1.2 & . & . & . & . & +.2 \\
\hline
\end{tabular}




\begin{tabular}{|c|c|c|c|c|c|c|c|c|c|c|c|c|c|c|c|}
\hline Successive $\mathrm{N}^{\circ}$ & 1 & 2 & 3 & 4 & 5 & 6 & 7 & 8 & 9 & 10 & 11 & 12 & 13 & 14 & 15 \\
\hline Galium aparine & . & . & . & 1.2 & 1.2 & . & +.2 & 1.2 & . & +.2 & . & . & . & . & + \\
\hline Rubus caesius & . & . & . & 1.2 & 1.2 & 2.2 . & +.2 & + & . & . & . & . & . & +.2 & \\
\hline Geranium robertianum & 1.2 & + & 1.2 & 1.2 & . & 1.2 & . & . & . & . & . & . & . & . & . \\
\hline Mycelis muralis & + & 1.1 & 1.1 & 1.1 & 1.2 & . & . & . & . & . &. & . & . &. & . \\
\hline Alliaria petiolata & + & 2.2 & 1.2 & + & . & . & . & . & . & . & . & . & . & . & . \\
\hline \multicolumn{16}{|l|}{ Ch. Calluno-Ulicetea } \\
\hline Agrostis capillaris & . & . & . & . & . & . & . & +.2 & . & . & +.2 & . & . & . & +.2 \\
\hline \multicolumn{16}{|l|}{ Others } \\
\hline Dryopteris carthusiana & + & + & . & . & . & 1.2 & +.2 & . & 1.2 & . & + & . & +.2 & +.2 & . \\
\hline Juncus conglomerates & . & . & . & . & . & + & +.2 & 1.2 & . & . & . & + & 1.2 & . & +.2 \\
\hline Carex ovalis & . & . & . & . & . & 1.2 & 2.2 & . & . & . & +.2 & 1.1 & . & . & +.2 \\
\hline Carex pallescens & . & . & . & . & . & 1.2 & . & . & +.2 & . & . & 1.2 & . & . & . \\
\hline Veronica chamaedrys & . & + & . & 2.2 & 1.1 & . & . & . & . & . & . & . & . & . & . \\
\hline Festuca rubra & & . & . & 1.2 & 2.2 & . & . & . & . & . & . & . & . & . & . \\
\hline Poa pratensis & . & . & . & 2.2 & 1.2 & . & . & . & . & . & . & . & . & . & . \\
\hline Oxalis acetosella & 1.2 & . & 1.2 & . & . & . & . & . & . & . & . & . & . & . & . \\
\hline Anthoxanthum odoratum & . & . & . & 1.2 & 1.2 & . & . & . & . & . & . & . & . & . & . \\
\hline Deschampsia caespitosa & . & 1.2 & 1.2 & . & . & . & . & . & . & . & . & . & . & . & . \\
\hline Carex hirta & . & . & . & . & . & . & +.2 & . & . & . & . & +.2 & 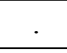 & & . \\
\hline Hypericum perforatum & . & + & . & . & . & . & . & . & . & . & . & . & . & . & + \\
\hline Hypericum montanum & . & . & . & + & + & . & . & . & . & . & . & . & . & . & . \\
\hline
\end{tabular}

Sporadic:

Trees and shrubs: Crataegus laevigata b 2 (+); Prunus avium c 6 (1.1); Ribes uva-crispa b 5 (1.2); Sambucus nigra c 3 (+); Ulmus glabra b 9 (1.1) Ch. Quercetea robori-petraeae: Festuca ovina 4 (1.2); Hieracium lachenalii 4 (+); Solidago virgaurea $4(+)$

Ch. Epilobietea: Rubus grabowskii 15 (2.2); Rubus radula $5(+)$;

Ch. Artemisietea: Geum urbanum 2 (+); Glechoma hederacea 5 (+.2); Humulus lupulus b, c 5 (1.2); Rubus pseudidaeus $6(+)$;

Others: Acinos arvensis 5 (1.2); Brachythecium rutabulum d 4 (1.2); Dryopteris dilatata 12 (+.2); Euhrynchium hians d 2 (1.2); Euphoprbia cyparisias 4 (+); Galium verum 4 (1.2); Luzula multiflora 12 (+.2); Myosoton aquaticum 3 (+); Plagiomnium affine d 2 (1.2); Rubus nessensis 8 (1.2); Vicia cassubica $11(+)$

Explanations: P - "Promno", C - "Czempiń", R - "Racot"

The Turkey oak coming from natural regeneration, covering no less than $2 \%$ of the area in the shrub layer, was usually found in substitute communities with pine tree stands in habitats of the mixed fresh forest type corresponding in the potential vegetation of central Wielkopolska to poor forms of oak-hornbeam forests or acidophilous oak forests (Tab. 3). Only in one place at 'Racot', the tree layer consisted of Betula pendula, and in two, the habitat was a fresh mixed coniferous forest. The age of tree stands at the time the relevés were made ranged from 59 to 87 years, which means that they reached the stage of a maturing or mature tree stand. They usually had a small proportion of additional species and a single layer. The most stable components of the shrub layer, apart from the Turkey oak, were Sorbus aucuparia, Quercus robur (at 'Promno' also Q. petraea) and Frangula alnus, and sometimes also Prunus serotina. The most popular species in the ground layer was Pleurozium schreberi accompanied, but only at 'Promno', by two other species characteristic of the class Vaccinio-Piceetea. An insignificant role was played by species diagnostic for other forest communities from the classes Quercetea roboripetraeae and Querco-Fagetea. Popular components of those phytocoenoses were plants representing the class of clearing communities, Epilobietea angustifolii, like Calamagrostis epigejos and various species of the genus Rubus, as well as Agrostis capillaris from the class Calluno-Ulicetea and Rumex acetosella from the class Koelerio-Ulicetea. In the group of accompanying species, the most important were the ferns Dryopteris carthusiana and $D$. dilatata. 


\begin{tabular}{|c|c|c|c|c|c|c|c|c|c|c|c|c|c|c|c|c|c|c|c|c|c|c|c|c|c|c|c|}
\hline$\stackrel{\searrow}{\sim}$ & $88 \pm$ & 8 & & in & ฉ & $\sqrt{v}$ & ¿্ণ & 0 & $a-6$ & & $\approx$ & $\vec{\sim}$ & & & & $\stackrel{\sim}{N}$ & & $\stackrel{+}{+}$ & & & & $\exists$ & & . & & & \\
\hline$\tilde{\sim}$ & $=8 \%$ & 8 & & $\infty$ & 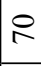 & f & ¿্ণ & $\simeq$ & $-a \infty$ & & $\stackrel{\infty}{\sim}$ & $\infty$ & & & $\begin{array}{c}y \\
\text { v }\end{array}$ & $\stackrel{m}{+}$ & $\stackrel{\sim}{i}$ & $\stackrel{m}{m}$ & + & & ָี & . & & & & & \\
\hline 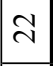 & $m 88$ & in & & $\triangleright$ & iి & 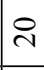 & ¿্ & $\simeq$ & $-a \infty 4$ & & $\stackrel{\infty}{\sim}$ & 2 & & & . & $\stackrel{m}{*}$ & $\stackrel{n}{i}$ & $\stackrel{+}{+}$ & & & . & . & + & $\cdot$ & & & . \\
\hline$\vec{\sim}$ & $=88$ & in & & 8 & 으 & 요 & ì & $\simeq$ & $-a \infty$ & & $\stackrel{\infty}{\sim}$ & \pm & & & & $\stackrel{m}{+}$ & $\begin{array}{l}n \\
i\end{array}$ & $\stackrel{+}{+}$ & & & $\cdot$ & $\exists$ & + & $\cdot$ & + & & . \\
\hline 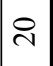 & $=8 \%$ & ? & & 8 & ণิ & $\stackrel{\sim}{~}$ & ¿ి & $\simeq$ & $-a \infty \psi$ & & $\stackrel{\infty}{\sim}$ & 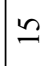 & & & & $\stackrel{m}{+}$ & $\stackrel{\sim}{i}$ & $\stackrel{\leftrightarrow}{+}$ & & & . & . & & . & & & . \\
\hline 9 & $=8 \%$ & in & & $\triangleright$ & 으 & ণे & ì & $\simeq$ & $-a \infty$ & & $\stackrel{\infty}{\sim}$ & $\stackrel{0}{-}$ & & & . & $\stackrel{m}{+}$ & $\stackrel{\sim}{i}$ & $\tilde{i}$ & . & & $\cdot$ & $\tilde{i}$ & $\stackrel{1}{=}$ & ה̃ & . & $\exists$ & . \\
\hline$\infty$ & $=8 \%$ & in & & 8 & P & \& & $\underset{\sim}{\stackrel{N}{~}}$ & $\simeq$ & $-a \infty \psi$ & & $\stackrel{\infty}{\sim}$ & $\vec{\sim}$ & & & & הָ & $\stackrel{\sim}{i}$ & $\stackrel{ナ}{+}$ & . & + & . & $\tilde{a}$ & & $\cdot$ & $=$ & & . \\
\hline$\simeq$ & $=8$ & 8 & & $R$ & in & 우 & ஓ्丶 & $\simeq$ & $-a \infty$ & & $\stackrel{\infty}{\infty}$ & $\Xi$ & & & & ç & + & $\stackrel{+}{+}$ & & & . & $\exists$ & & . & . & $\exists$ & . \\
\hline 으 & 응 & in & & in & $\infty$ & o & ¿ั & $\simeq$ & - & & $n$ & ন & & & . & $\widetilde{N}$ & + & $\stackrel{+}{+}$ & . & + & $\cdot$ & $\tilde{m}$ & . & $\cdot$ & $=$ & & . \\
\hline 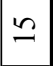 & 응 & 8 & & fo & 8 & in & ¿্ণ & $\simeq$ & $-\infty \infty 0$ & & $n$ & $\stackrel{0}{-}$ & & & & $\vec{i}$ & + & $\stackrel{ナ}{+}$ & . & + & . & ָ̃ & + & $\cdot$ & . & & . \\
\hline$\Xi$ & 응 & 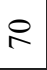 & & in & f & in & సి & $\simeq$ & $-\infty \infty$ & & $n$ & $\infty$ & & & & $\vec{i}$ & + & $\stackrel{+}{+}$ & . & + & . & $\vec{i}$ & + & $\cdot$ & + & & . \\
\hline 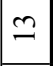 & 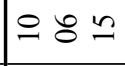 & 若 & 0 & \& & ণ & f & $\stackrel{i}{~}$ & $\simeq$ & NOO & 点 & $\infty$ & $\simeq$ & & & $\begin{array}{l}\dot{y} \\
c \\
c\end{array}$ & $\tilde{n}$ & . & $\stackrel{*}{*}$ & & & $\stackrel{*}{i}$ & . & . & $\cdot$ & . & & \\
\hline$\simeq$ & $\circ 8 \div$ & : & in & in & in & ণ & ¿े & $\simeq$ & not & 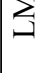 & $\infty$ & $\mathscr{0}$ & & & $\vec{i}$ & ָூ & + & $\stackrel{*}{*}$ & . & & $\stackrel{*}{i}$ & . & . & . & . & & $\exists$ \\
\hline$=$ & 응 & & & i & $\infty$ & f & ¿ & $\simeq$ & $-a r \cdot$ & & రิ & $\approx$ & & & & ri & $=$ & $\stackrel{+}{+}$ & & & . & $\tilde{i}$ & & . & $\exists$ & & . \\
\hline 으 & $\circ 8 \div$ & 8 & & in & $\infty$ & 8 & ¿ి & $\simeq$ & $-a r \cdot$ & & రु & $=$ & & & 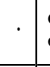 & $\stackrel{\sim}{n}$ & $=$ & $\stackrel{+}{+}$ & . & + & . & $\stackrel{\sim}{i}$ & & . & & & . \\
\hline$a$ & 으 8 ㄴ & 8 & & 8 & 8 & 우 & ¿্ & $\simeq$ & $-a r r$ & & ชิ & $\infty$ & & & & $\stackrel{\sim}{n}$ & . & $\stackrel{+}{+}$ & . & & . & $\vec{a}$ & . & $\exists$ & $\exists$ & & . \\
\hline$\infty$ & 으 8 ㄴ & 8 & & in & 8 & \& & ¿্ণ & $\simeq$ & $-a r \cdot$ & & రु & $=$ & & & & $\tilde{n}$ & + & $\stackrel{ナ}{+}$ & & & . & $\stackrel{\sim}{i}$ & + & . & & & . \\
\hline$r$ & 응 & 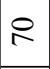 & & 웅 & 8 & ? & ì & $\simeq$ & $-a r \cdot-$ & & ชิ & 2 & & & . & $\tilde{n}$ & $=$ & $\exists$ & . & & . & $\exists$ & + & $\stackrel{+}{+}$ & & & . \\
\hline 6 & $=8 \backsim$ & $\infty$ & & 요 & 8 & 운 & iి & $a$ & $n-6$ ad & & 8 & $\stackrel{+}{\sim}$ & & & & $\underset{N}{~}$ & + & $\ddot{n}$ & 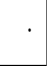 & & . & $\tilde{i}$ & + & $\cdot$ & + & & . \\
\hline in & $=8 \backsim$ & in & & $\infty$ & 운 & 8 & 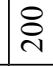 & 2 & $n \rightarrow-\pi$ & & 8 & $\approx$ & & & & ry & + & $\stackrel{m}{+}$ & & & . & $\stackrel{\sim}{\sim}$ & & . & . & & . \\
\hline$\nabla$ & $=8 \div$ & R & & in & 8 & $\infty$ & ì & 2 & 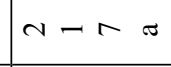 & & 8 & $\approx$ & & & & ָู & & $\stackrel{+}{+}$ & & & . & $\stackrel{1}{=}$ & + & . & + & & . \\
\hline$m$ & $=8 \div$ & 8 & & $\triangleright$ & 8 & $\infty$ & ণิ & 2 & $n \sim+u$ & & in & $\stackrel{+}{\sim}$ & & & & הֶ & & $\stackrel{+}{+}$ & & & . & $\stackrel{n}{i}$ & & . & & & . \\
\hline$\sim$ & $=8 \backsim$ & 8 & & in & $\infty$ & 운 & ஓ্ণ & 2 & 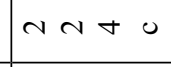 & & in & 9 & & & & $\stackrel{\text { I }}{\mathrm{N}}$ & & $\stackrel{\bullet}{+}$ & & & . & $\exists$ & + & . & . & & . \\
\hline- & $=8 \backsim$ & ir & & 8 & in & ฉ & 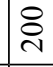 & 2 & $a v+u$ & & के & ণิ & & & & $\stackrel{\text { in }}{n}$ & & $\stackrel{+}{+}$ & & & & . & + & . & & & . \\
\hline 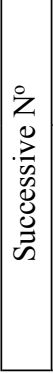 & 胥 & 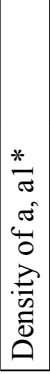 & 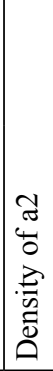 & 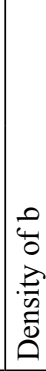 & \begin{tabular}{|l}
0 \\
4 \\
\\
$\dot{0}$ \\
0 \\
0 \\
0
\end{tabular} & $\begin{array}{l}\tau \\
\tilde{0} \\
0 \\
\dot{0} \\
0 \\
0\end{array}$ & 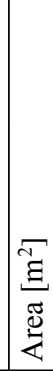 & : & 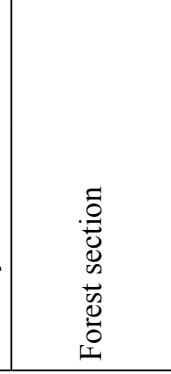 & 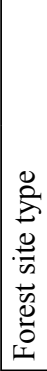 & 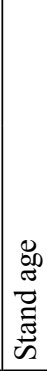 & 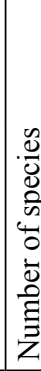 & 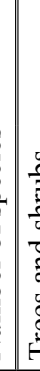 & & 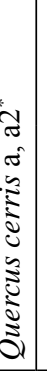 & 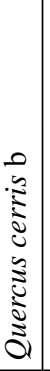 & 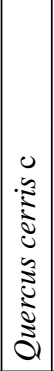 & 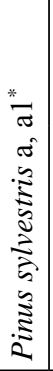 & 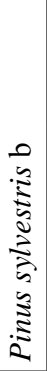 & 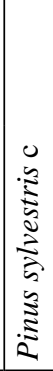 & 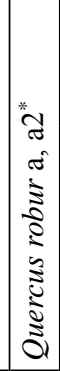 & 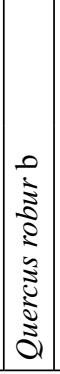 & 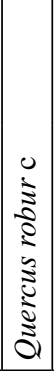 & 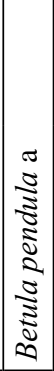 & 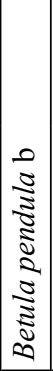 & $\begin{array}{l}\tilde{\sigma} \\
\tilde{u} \\
\tilde{z} \\
\tilde{z} \\
\tilde{z} \\
\end{array}$ & 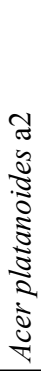 \\
\hline
\end{tabular}




\begin{tabular}{|c|c|c|c|c|c|c|c|c|c|c|c|c|c|c|c|c|c|c|c|c|c|c|c|c|c|c|c|c|c|c|}
\hline \multicolumn{2}{|c|}{$\stackrel{4}{4}$} & $\cdot$ & & & & & & . & & . & $\cdot$ & & & & & & & & & & + & $\cdot$ & & & & & + & & & \\
\hline ते & $\cdot$ & $\exists$ & + & + & + & & & $\exists$ & + & . & . & & . & & & & & & & & $\cdot$ & $\cdot$ & $\exists$ & & $\cdot$ & & $\cdot$ & 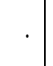 & & \\
\hline ส & $\cdot$ & $\exists$ & . & 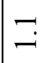 & & & & & & & & & $\cdot$ & $\exists$ & & & & & . & & . & & & & $\exists$ & + & & & & \\
\hline$\vec{\sim}$ & $\cdot$ & $\stackrel{\sim}{\sim}$ & $\stackrel{\sim}{i}$ & & + & $\tilde{N}$ & & & & . & . & & . & & & & & & $\cdot$ & & . & & & & $\cdot$ & & & & & \\
\hline i) & & $\begin{array}{l}\tilde{\lambda} \\
\end{array}$ & तี & 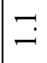 & . & & + & & & . & . & & . & . & + & . & & & 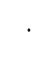 & & . & $\cdot$ & & & $\cdot$ & + & . & . & & \\
\hline 2 &. & $\begin{array}{c}\sim \\
m\end{array}$ & $\stackrel{\sim}{i}$ & $\exists$ & . & $\exists$ & & & & . & . & & . & . & + & $\exists$ & + & & 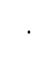 & & . & . & & & . & & $\cdot$ & $\cdot$ & & \\
\hline$\stackrel{\infty}{-1}$ & $\cdot$ & 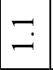 & & $\exists$ & $\cdot$ & ָ̃ & તี & $\stackrel{\sim}{i}$ & & $\exists$ & . & & $\cdot$ & $\exists$ & & & & & $\cdot$ & 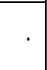 & $\cdot$ & . & & & $\cdot$ & & $\cdot$ & & & \\
\hline$=$ & & $\rightleftarrows$ & & & . & & $\stackrel{\text { I }}{~}$ & 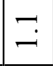 & & $\cdot$ & + & & . & . & + & . & & & . & $\cdot$ & . & $\cdot$ & $\exists$ & & . & & $\cdot$ & & & \\
\hline- &. & $\vec{\sim}$ & . & & ${ }^{\circ}$ & & $\stackrel{\sim}{\sim}$ & + & & $\exists$ & + & & . & . & & . & & & . & & . & . & . & + & . & & $\cdot$ & $\cdot$ & & \\
\hline$\simeq$ & $\cdot$ & $\vec{i}$ & . & $\exists$ & + & & . & + & & + & & & . & . & & . & & & $\cdot$ & $\cdot$ & $\cdot$ & . & & & & & & & & \\
\hline \pm &. & $\vec{i}$ & & + & . & & . & & & + & + & & . & . & & . & & & . & $\cdot$ & $\cdot$ & . & . & & . & & $\cdot$ & & & \\
\hline$\approx$ & $\cdot$ & + & . & + & . & $\stackrel{m}{m}$ & + & $\tilde{i}$ & & $\cdot$ & . & & . & . & & . & & & . & 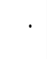 & 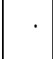 & . & & & $\cdot$ & & . & . & & \\
\hline$\simeq$ & $\cdot$ & $\cdot$ & + & + & . & $\stackrel{m}{n}$ & $\stackrel{\text { I }}{~}$ & in & & $\cdot$ & . & & . & & + & . & & & . & . & $\cdot$ & . & & & & & & & & \\
\hline$=$ &. & $\vec{i}$ & + & + & . & 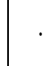 & . & + & & $\vec{i}$ & . & & . & . & & . & & & . & . & . & . & . & & . & & . & . & & \\
\hline 의 & $\cdot$ & $\begin{array}{l}\tilde{N} \\
\mathrm{i}\end{array}$ & + & 二 & . & + & . & . & & . & . & & . & . & & . & & & . & . & & . & & & $\cdot$ & & . & . & & \\
\hline a & $\exists$ & $\exists$ & . & + & . & & . & . & & $\cdot$ & + & & . & & & . & & & . & . & . & . & & & . & & & . & & \\
\hline$\infty$ & $\cdot$ & $\exists$ & + & & + & & . & $\cdot$ & & + & . & & . & & & & & & . & $\cdot$ & . & . & & & . & & & & & \\
\hline r &. & $\exists$ & & & & & . & . & & $\rightleftarrows$ & . & & + & & & . & & & . & 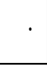 & $\cdot$ & . & & & . & & & . & & \\
\hline ○) & $\cdot$ & $\cdot$ & . & $\stackrel{\text { I }}{3}$ & + & $\tilde{i}$ & + & . & & & + & & . & . & & . & . & $\exists$ & . & . & . & + & & & . & & . & . & & + \\
\hline in & $\cdot$ & ה & + & . & . & $\stackrel{m}{n}$ & + & $\stackrel{1}{I}$ & & $\cdot$ & . & + & . & & & . & & + & . & . & . & . & & & . & & & & & \\
\hline t &. & $\stackrel{\sim}{\sim}$ & & $\stackrel{\text { ִָ }}{ }$ & + & $\ddot{m}$ & + & $\cdot$ & & . & . & & . & . & & . & & & . & 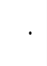 & . & . & . & & . & & . & + & & \\
\hline$m$ & . & . & & $\exists$ & . & $\exists$ & + & $\cdot$ & & . & . & กิ & . & . & & . & & & $\exists$ & + & & . & & & . & & . & & & \\
\hline$\sim$ & & $\rightleftarrows$ & & . & . & & . & . & & . & & $\tilde{i}$ & . & & & & + & & . & . & . & . & & & . & & & & 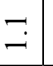 & \\
\hline- & . & $\vec{i}$ & & + & . & 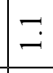 & + &. & & $\cdot$ & $\cdot$ & ş & . & & & & + & & & & & & & & . & & & . & & \\
\hline 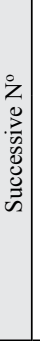 & 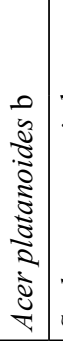 & 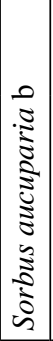 & 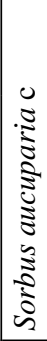 & 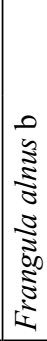 & 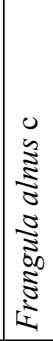 & 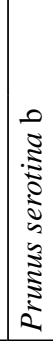 & 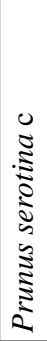 & 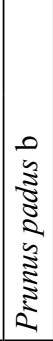 & 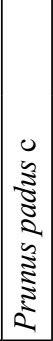 & 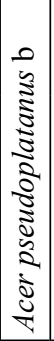 & 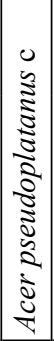 & 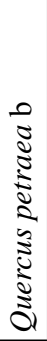 & 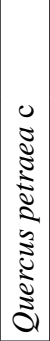 & 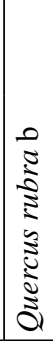 & 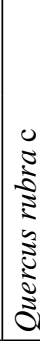 & 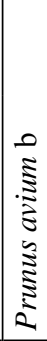 & 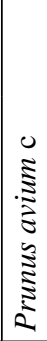 & 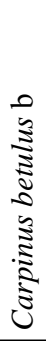 & 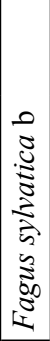 & 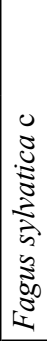 & 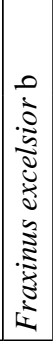 & 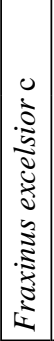 & 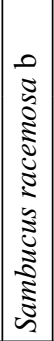 & 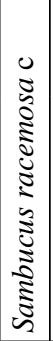 & 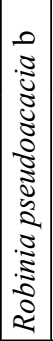 & 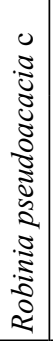 & 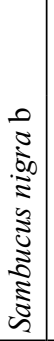 & 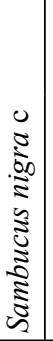 & 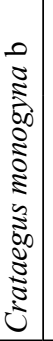 & 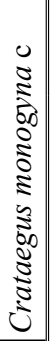 \\
\hline
\end{tabular}




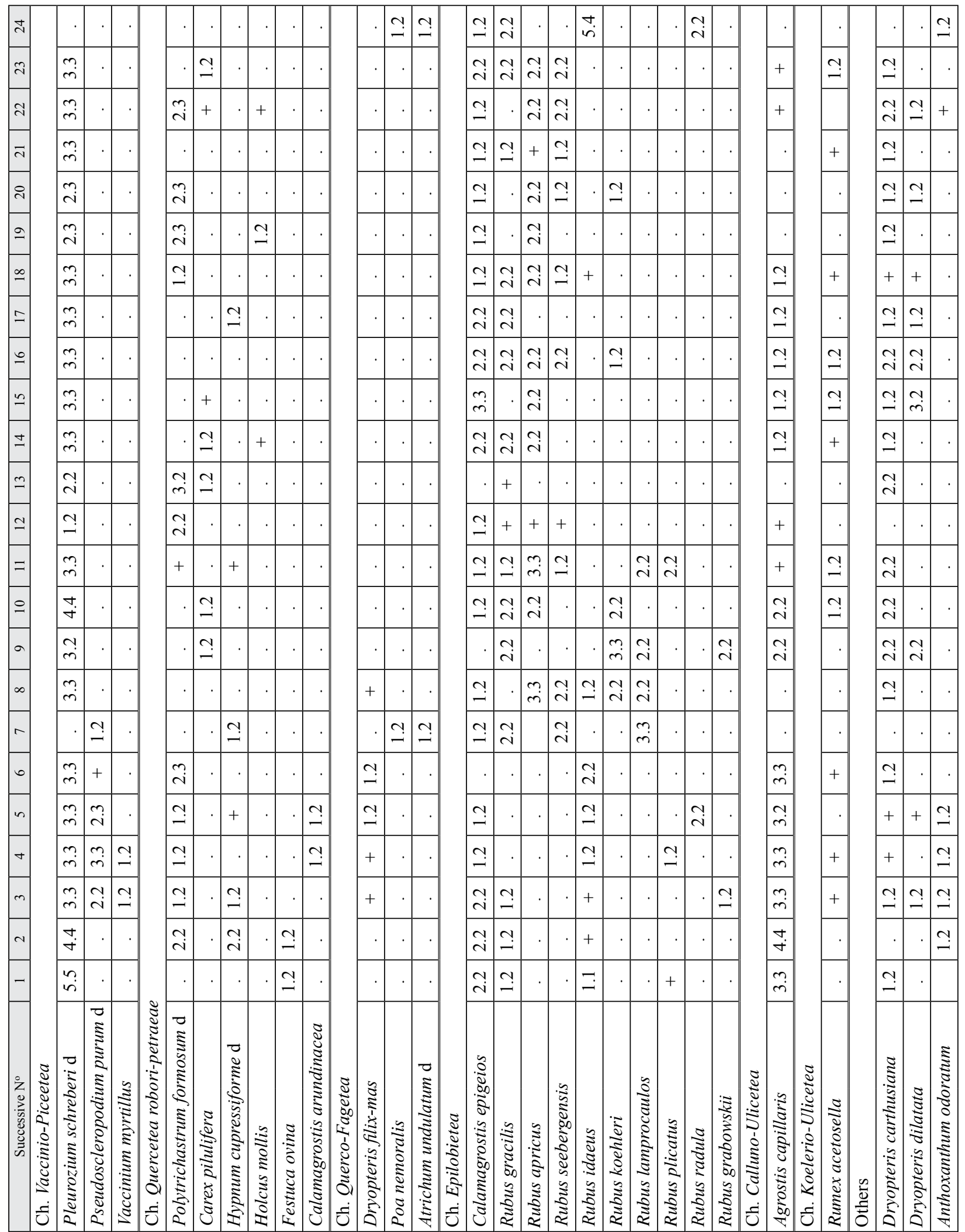




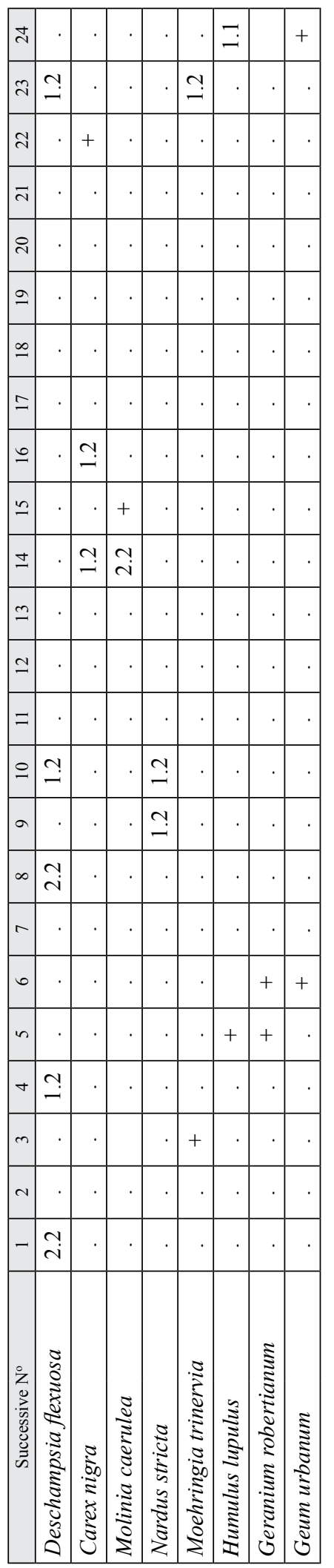

\section{Discussion}

In the literature on the naturalisation of alien oaks in central Europe, most attention has so far been paid to the establishment and dispersal of Quercus rubra (Vor 2005; Straigyté and Žalkauskas 2012; Chmura 2013, 2014; Gazda 2013; Major et al. 2013; Woziwoda et al. 2013, 2014; Myczko et al. 2014; Dyderski 2015). This is due to the fact that in many regions, this has been the broad-leaf tree of alien origin cultivated most frequently, and its capacity for spontaneous reproduction and expansion in forests has long been known. Although the dynamic tendencies of $Q$. rubra have been an object of study in a variety of environmental conditions and over large areas, to this day, its capacity for a spontaneous penetration of plant communities has not been satisfactorily explained (Woziwoda et al. 2013, 2014; Danielewicz and Wiatrowska 2014).

Because of its little significance for forestry and the small introduction range, the Turkey oak has not been such an attractive research object. This paper presents several pieces of evidence showing $Q$. cerris to have a greater invasive potential, at the scale of a few sites, which suggests that the penetration of the woodland environment by this species could be wider if its cultivation was more widespread.

As has been demonstrated, the most probable sources of seeds for the dispersal of the Turkey oak are individual, small tree stands established more than 100 years ago in habitats suitable for the cultivation of native oak species. At 'Racot', the distance separating the parent trees from the farthest localities of their young generation is almost $1.5 \mathrm{~km}$. This is the distance over which acorns of Quercus robur and Q. petraea are carried by jays (Ouden et al. 2005). But, an intriguing question is whether the young Turkey oaks at 'Promno' found that some $2.5 \mathrm{~km}$ from old trees of this species can be proof of a greater distance over which its acorns are dispersed via zoochory. Among interesting issues connected with this is the chronology of $Q$. cerris entering new localities. Is it a gradual and steady process, or rather one intensive in only some periods when acorns develop most abundantly? And, how does it affect the fruiting of the other oak species growing in the same area, but producing fruits at a different time? Answers to those and similar questions can be supplied by detailed studies of the age structure of the natural regeneration and biology 
of the Turkey oak in secondary localities and the role of zoochory in its dispersal.

An analysis of the size characteristics of the Turkey oak specimens from self-sown seedlings in the study area makes it possible to state that this species is not only established, but locally meets all the criteria by which it should be classified as belonging to the group of neophytes or species attaining the stage of naturalisation (cf. Faliński 1968, 1969; Richardson et al. 2000; Pyšek et al. 2004; Tokarska-Guzik 2012). Such plants are capable of regular (rather than sporadic) reproduction and of producing permanent secondary generations the existence of which does not depend on direct human activity.

\section{Conclusions}

Quercus cerrisis capable of a natural renewal in a woodland environment in Poland. In the light of the various definitions concerning the synanthropisation of the plant cover, it can be problematic to regard the Turkey oak as an invasive plant in forests. While it penetrates forest communities spontaneously and has a stable position in the undergrowth or even lower tree layers in them, those phytocoenoses greatly depart from the permanent natural communities in terms of structure and floristic composition. The dispersal of $Q$. cerris in them is similar to that of other oaks species, often renewing under the canopy of pine tree stands (Mosandl and Kleinert 1998; Pigan and Pigan 1999; Gómez 2003; Sokołowski and Paluch 2003; Gniot 2007). In many cases, this reflects the regeneration of forest communities in which native plants can be accompanied by alien species. If we assume, following Sukopp's (1962) conception, that a neophyte appearing in such 'unsaturated' phytocoenoses does not push out native species, and the proportion of the Turkey oak to the already present community components, according to Faliński's (1968) criteria, can be called compensatory, at least for the time being, then classifying it as an invasive plant is disputable in terms of definitions emphasising the threat to biological diversity posed by such plants. However, if we were to use the criterion of the rate of dispersal of alien species - over a distance longer than $100 \mathrm{~m}$ in a period shorter than 50 years (Richardson et al. 2000), then Q. cerris would have the status of an invasive plant.

\section{References}

Bozano M., Turok J. 2003. Mediterranean Oak Networks. Reports of second meeting, 2-4 May 2002, Gozo, Malta. International Plant Genetic Resources Institute, Rome.

Browicz K. 1982. Chorology of trees and shrubs in South-West Asia and adjacent regions. Vol. 1. Polish Scientific Publishers, Warszawa.

Chmura D. 2013. Impact of alien tree species Quercus rubra L. on understory environment and flora: a study of Silesian Upland (Southern Poland). Polish Journal of Ecology, 61 (3), 431-442.

Chmura D. 2014. Phytocenotic characteristics of secondary forest communities with contribution of Quercus rubra L. in the Silesian Upland. Acta Botanica Silesiaca, 10, 17-40.

Danielewicz W., Kiciński P., Antosz Ł. 2014. Turkey oak (Quercus cerris L.) in Polish forests. Acta Scientiarum Polonorum. Silvarum Colendarum Ratio et Industria Lignaria, 13 (2), 5-22.

Danielewicz W., Maliński T. 2003. Alien tree and shrubs species in Poland regenerating by self-sowing. Rocznik Dendrologiczny, 51, 205-236.

Danielewicz W., Wiatrowska B. 2014. The most invasive tree and shrub species in Polish forests (in Polish). Peckiana, 9, 59-67.

Dyderski M.K. 2015. Spread of Northern Red Oak in the Wielkopolski National Park (in Polish). Department of Game Management and Forestry Protection of Poznań University of Life Sciences, Poznań.

Faliński J.B. 1968. Stages of neophytism and the relation of neophytes to other components of the community (in Polish). Materiaty Zakładu Fitosocjologii Stosowanej UW, 25, 15-31.

Faliński J.B. 1969. Neophytes and neophytism (in Polish). Ekologia Polska Seria B, 15 (4), 337-355.

Gazda A. 2013. Distribution of alien tree species in various forest communities of southern Poland (in Polish). Zeszyty Naukowe Uniwersytetu Rolniczego im. H. Koltataja w Krakowie, 512 (389), 1-117.

Gniot M. 2007. The oak succession in the pine stand in the coniferous forest habitats (in Polish). Sylwan, $151(5), 60-72$.

Gómez J.M. 2003. Spatial patterns in long-distance dispersal acorns of Quercus ilex acorns by jays in a heterogeneous landscape. Ecography, 26, 573-584. 
Jalas J., Suominen J. 1976. Atlas Florae Europaeae. Distribution of Vascular Plants in Europe. Vol. 3. Salicaceae to Balanophoraceae. The Committee for Mapping the Flora of Europe and Societas Biologica Fennica Vanamo, Helsinki.

Juwa M.M. 1968. Turkey oak and other alien tree species in Promno Forest District (Czereniejewo Forest Inspectorate) (in Polish). Department of Forest Botany of Agricultural University, Poznań.

Kliczkowska A., Zielony R., Czępińskia-Kamińska D., Kowalkowski A., Sikorska E., Krzyżanowski A., Cieśla A., Czerepko J. 2004. Habitat basics of silviculture. Appendix to the rules of silviculture (in Polish). Ośrodek Rozwojowo-Wdrożeniowy Lasów Państwowych w Bedoniu, Warszawa.

Major K.C., Nosko P., Kuehne C., Campbell D., Bauhus J. 2013. Regeneration dynamics of non-native northern red oak (Quercus rubra L.) populations as influenced by environmental factors: A case study in managed hardwood forests of southwestern Germany. Forest Ecology and Management, 291, 144-153.

Menitski Yu. L. 1984. Asia’s Oaks (in Russian). Nauka, Leningrad.

Meusel H., Jäger E., Weinert E. 1965. Comparative chorology of the Central European Flora (in German). Gustav Fischer Verlag, Jena.

Mosandl R., Kleinert A. 1998. Development of oaks (Quercus petraea (Matt.) Liebl.) emerged from bird-dispersed seeds under old-growth pine (Pinus sylvestris L.) stands. Forest Ecology and Management, 106, 33-44.

Myczko Ł., Dylewski Ł., Zduniak P., Sparks T.H., Tryjanowski P. 2014. Predation and dispersal of acorns by European Jay (Garrulus glandarius) differs between a native (Pedunculate Oak Quercus robur) and an introduced oak species (Northern Red Oak Quercus rubra) in Europe. Forest Ecology and Management, 331, 35-39.

Olaczek R. 1972. Anthropogenic degeneration forms of forest communities in the agricultural landscape of Polish lowland (in Polish). Wydawnictwo Uniwersytetu Łódzkiego, Łódź.

Olaczek R. 1974. Directions of forest communities degeneration and their research methods (in Polish). Phytocoenosis, 3 (3/4), 179-190.

Ouden J., Jansen P.A., Smit R., Forget P.M., Lambert J.E., Hulme R.E., Vander Wall S.B. 2005.
Jays, mice and oaks: predation and dispersal of Quercus robur and Q. petraea in North-western Europe. In: Seed fate: predation, dispersal and seedling establishment (eds.: P.M. Forget, J. Lambert, S.B. Vander Wall). CABI Publishing, Wallingford, 223-240.

Pigan I., Pigan M. 1999. Natural regeneration of pedunculate oak in pine stands (in Polish). Sylwan, 143 (9), 23-30.

Preston C.D., Pearman D.A., Dines T.D. 2002. New atlas of the British and Irish flora. Oxford University Press, Oxford.

Pyšek P., Richardson D.M., Rejmánek M., Webster G.L., Willamson M., Kirschner J. 2004. Alien plants in checklist and floras: towards better communication between taxonomists and ecologists. Taxon, 53 (1), 131-143.

Richardson D.M., Pyšek P., Rejmánek M., Barbour M.G., Panetta F.D., West C.J. 2000. Naturalization and invasion of alien plants: concepts and definitions. Diversity and Distribution, 6, 93-107.

Sokołowski A., Paluch R. 2003. Expansion of oak in pine stands (in Polish). Las Polski, 2, 22-23.

Straigyté L., Žalkauskas R. 2012. Effect of climate variability on Quercus rubra phenotype and spread in Lithuanian forests. Dendrobiology, 67, 79-85.

Sukopp H. 1962. Neophytes in natural plant communities in Central Europe (in German). Berichte der Deutschen Botanischen Gesellschaft, 75 (6), 193-205.

Tokarska-Guzik B., Dajdok Z., Zając M., Zając A., Urbisz A., Danielewicz W., Hołdyński C. 2012. Alien plants in Poland with particular reference to invasive species (in Polish). Generalna Dyrekcja Ochrony Środowiska, Warszawa.

Vor T. 2005. Natural regeneration of Quercus rubra L. (red oak) in Germany. Neobiota, 6, 111-123.

Woziwoda B., Kopeć D., Witkowski J. 2013. The negative impact of intentionally introduced Quercus rubra L. on a forest community. Acta Societatis Botanicorum Poloniae, 83 (1), 39-49.

Woziwoda B., Potocki M., Sagan J., Zasada M., Tomusiak R., Wilczyński S. 2014. Commercial forestry as a vector of alien tree species - the case of Quercus rubra L. introduction in Poland. Baltic Forestry, 20 (1), 131-141. 